\title{
Funciones del sistema ideológico de la excelencia en el espacio académico.
}

\author{
Functions of the ideological system of excellence in the \\ academic field
}

Antonio Benedito Casanova1

José Beltrán Llavador²

A Jorge Wagensberg, in memoriam

\section{RESUMEN}

En nuestras universidades públicas estamos asistiendo a un progresivo proceso de cambio que se ha acelerado en las dos últimas décadas y que presenta no pocas paradojas. Nuestro análisis parte del intento de clarificar, en este contexto, una llamativa antinomia entre los discursos de diferentes agentes sociales universitarios que alertan contra la precariedad, la falta de recursos y el deterioro de nuestro orden institucional universitario y los discursos de esos mismos agentes, obnubilados por el aumento de la calidad, la excelencia y la productividad de las instituciones universitarias a las que pertenecen. Siempre llama la atención el aparente hecho de que se haga más con menos y que se ponga todo en manos de la gestión y no de la producción de recursos materiales como respuesta inteligente a las contradicciones, sobre todo cuando la propia virtud de la excelencia supone como incuestionable una serie de medios, bienes y capitales previamente dados. La compleja crisis institucional que atraviesan las universidades, vinculable a la de nuestras sociedades, viene acompañada de una recurrente apelación a la gobernanza numérica como única garante de una posible salida de tal crisis. La presente reflexión se sitúa en una investigación crítica acerca de las formas de cambio de la universidad contemporánea, tomando como hilo conductor la evolución de sus específicos modos de legitimación, manifestada en un sistema de discursos acerca de la universidad, producido tanto en su interior como en su exterior. Sostenemos que la sustitución del gobierno autónomo de la universidad por su gobernanza matemática pone en circulación los tres procesos clave de cualquier funcionamiento de un campo ideológico, correspondientes a sus dimensiones imaginaria, simbólica y real: la mitificación, la mistificación y la fetichización.

\section{PALABRAS CLAVE}

Excelencia académica, cambio social, ideologías, universidades, sistema de discursos, rankings universitarios.

\footnotetext{
1 Universitat de València. antonio.benedito@uv.es

2 Universitat de València. jose.beltran@uv.es
} 


\section{ABSTRACT}

We are witnessing a progressive process of change in our public universities that has accelerated in the last two decades and presents many paradoxes. Our analysis comes from the attempt to clarify, in this context, a striking antinomy between the discourses of different university social agents that warn against precariousness, lack of resources and the deterioration of our university institutional order and the discourses of those same agents, blinded for the increase in quality, excellence and productivity of the university institutions to which they belong. We must pay attention to the apparent fact that more is always done with less and that everything is put in the hands of the management and not of the production of material resources as an intelligent response to contradictions, especially when the virtue of excellence supposes unquestionable a series of means, goods and capital previously given. The complex institutional crisis facing universities, linked to that of our societies, is accompanied by a recurrent appeal to numerical governance as the only guarantor of a possible way out of such a crisis. The present reflection is placed in a critical investigation about the forms of change of the contemporary university, taking as a thread the evolution of its specific modes of legitimation, manifested in a system of discourses about the university, produced both inside and outside the university. We argue that the substitution of the autonomous government of the university for its mathematical governance puts into circulation the three key processes of any functioning of an ideological field, corresponding to its imaginary, symbolic and real dimensions: mythification, mystification and fetishization.

\section{KEYWORDS}

Academic excellence, social change, ideologies, universities, discourse system, university rankings. 
Un sistema de indicadores sintéticos para un Sistema Universitario Español (Pérez y Aldás, 2018): la propuesta de este proyecto nacional de investigación [ISSUE] no puede ser más clara. Por un lado, se trabaja en la dirección de contribuir a la configuración y consolidación de un determinado tipo de objeto, el Sistema Universitario Español. Por otra parte, se acentúa la naturaleza sistémica del programa. Las novedosas aportaciones de este Informe de la Fundación BBVA y el Ivie, en su sexta edición, referentes 1] a la posibilidad de que los usuarios puedan diseñar rankings personalizados según preferencias, es decir, según objetivos y criterios múltiples, 2] a la evolución del rendimiento universitario y 3] a la comparación entre universidades públicas y privadas, no puede hacernos obviar el fundamental giro epistemológico que representa el diseño de este sistema integral de cuatro rankings.

Ya no se trata meramente de ordenar universidades sin cumplir las condiciones formales de cualquier clasificación, como hacen los habituales rankings internacionales del Times y de la universidad de Shanghai. Se trata más bien de poder tomar medidas a las diferentes actividades que componen el proceso universitario [docencia, investigación e innovación] satisfaciendo los requisitos formales y materiales de un sistema de clasificación natural. El paso de una modelización analítica y práctica a otra sistémica y teórica no puede ser más significativo del singular momento histórico que estamos viviendo en nuestras universidades públicas. Los rankings habitualmente usados por ciudadanos, gobernantes y gestores universitarios para ordenar y comparar universidades de rango mundial [WCU: World Class Universities] son analíticos, porque toman a las universidades como entidades aisladas, que disponen de una serie de características propias [Salmi, 2009]. Y son prácticos, porque desde un determinado modelo ideal (singular, individual] devienen visibles/presentes el resto de universidades de categoría excelente. De algún modo, la idea única del bien supremo (Harvard] ilumina, situándolas, al resto de las privilegiadas universidades. Es decir, desde un lugar de invisibilidad se invisibiliza a la mayoría de universidades del mundo. La modelización española a la que hemos hecho referencia es sistémica, porque toma a cada universidad como un sistema de actividades y al conjunto de las universidades también como un sistema. Y es teórico, porque ya no parece existir ninguna instancia invisible desde la que se observa, sino que la totalidad de universidades son visibles y comparables; es más, se añade la conceptualización métrica a la comparativa. Podemos saber "cuánto es más productiva" una universidad que otra. Lo que media entre un operacionalizado fin (rendimiento) y un sistema de conceptos clasificatorios es un preciso objeto matemático.

A pesar del avance que suponen estos nuevos sistemas de clasificación no podemos dejar de hacernos los siguientes interrogantes: si los rankings internacionales más usados nos remiten a la adquisición de recursos humanos y financieros y el sistema de rankings ISSUE nos dirige hacia la producción de resultados, ¿son medibles y comparables las universidades por sus procesos de adquisición y depredación de recursos o por sus procesos de producción de utilidades? Si en las primeras clasificaciones se presentan las relaciones de adquisición como relaciones de producción, ¿se presentan en las segundas las relaciones de producción como de adquisición? La exigencia de transparencia y de rendición de cuentas de las universidades parece conducirlas al tratamiento de las mismas como entidades de producción de valor y de plusvalor. No abordaremos, pues, los diferenciados procesos de liberalización, comercialización, mercantilización y privatización que afectan al campo social universitario. Tampoco analizaremos este campo como un espacio de fuerzas sociales y de luchas por conservar o transformar esas relaciones de fuerza [Bourdieu, 2016]. Nos centramos más bien en los modos como se nos presentan los hechos a través de los rankings, las contradicciones que detectamos, los modos 
en que se toma conciencia de esos hechos y las formas supuestamente científicas que se están produciendo para dar cuenta de los hechos, de los discursos y de las paradojas de ambos.

En lo que sigue nuestra propuesta se desarrollará en tres apartados. El primero se dedica a la observación de la clasificación de las universidades de rango mundial a través de los principales rankings internacionales. Aquí prestaremos especial atención a la experiencia española a partir de la construcción reciente de un sistema propio de indicadores sintéticos y a la posición de las universidades españolas entre las WCU. Se pondrá énfasis en algunas características de la nueva ortodoxia que supone la irradiación de la propia idea de "universidad de rango mundial", así como en la necesidad de cuestionarla por las antinomias que presenta. Esbozaremos los lineamientos de la caracterización del paradigma de la "excelencia" en tanto que mutación del campo ideológico que gobierna nuestras universidades y que será, a su vez, objeto de cambio.

En el segundo apartado escogemos "excelencia" como palabra clave asociada a la etiqueta de World Class Universities. Argumentamos nuestra decisión en base a su complementariedad funcional y expresiva con otros conceptos [gobernanza, equidad, calidad,] que los autores hemos explorado en trabajos previos sobre educación superior [Joshi y Paivandi 2015; Paivandi y Joshi 2016; Georgios, Joshi y Paivandi 2017). Pondremos en relación el término "excelencia" con las tres nociones mencionadas para explicar el carácter histórico del campo ideológico que analizamos. Y haremos un hincapié especial en la transformación de la noción de excelencia y la reemergencia de la idea de rendimiento

Finalmente abordaremos de qué modo la transformación del paradigma de la "excelencia" en el de la "eficacia" es la propuesta supuestamente científica para abordar las antinomias de la cultura de la excelencia.

Nuestro argumento puede ser sintetizado del siguiente modo: los sistemas de clasificación universitarios tienen a su base un sistema de representaciones sociales que siguen en la línea apuntada por Durkheim y Mauss de alejarse de las organizaciones de la estructura social. Su aportación específica consiste en contribuir a constituir un determinado ente artificial autónomo, el propio sistema universitario, con su propia lógica productiva. En la construcción de los hechos de ese sistema se producen y manifiestan contradicciones que llevan a las clasificaciones a complejizarse. En el abordaje sistémico de las universidades se llevan a cabo tres operaciones. La de fetichización, que cumple la función de presentar lo que es como no siendo. La de mitificación, cuyo objetivo es ocultar que no podamos acceder a lo que es. Y la de mistificación, que da razón del ser del no ser. De este modo, la construcción de ese sistema se refuerza ante posibles y eventuales cuestionamientos y problematizaciones.

\section{EL FETICHISMO DE LOS RANKINGS UNIVERSITARIOS: DIMENSIÓN REAL DE LA IDEOLOGÍA ACADÉMICA}

La excelencia, entre sus dos diferentes acepciones [tratamiento de una dignidad o calidad extrema], es una palabra para iluminar lo que no brilla con suficiente luz propia. [17]

1 Los aforismos que acompañan a cada epígrafe son de Jorge Wagensberg y proceden de "La excelencia en aforismos", publicado en la sección Tribuna Libre de El País, 8 de septiembre de 2017. Los números recogen la numeración del autor en esta publicación. A Wagensberg [2017] le debemos también en buena medida la estructuración lógica de este trabajo: pretendemos hacer observables y comprensibles los sistemas de clasificación universitarios, diferenciando entre objetos reales ideológicos y objetos de conocimiento. Esta separación entre método ideológico y método sociológico es la ya clásica apuntada por E. Durkheim [1974). En la misma línea somos deudores del trabajo de E.Trías [1975) sobre el concepto de "ideología" en K. Marx. Un reciente estudio de C. Ramas [2018] señala la necesidad de distinguir en el análisis marxiano entre fetichismo y mistificación. 
Fernández Enguita,[2016] ha publicado un estudio sobre las transformaciones educativas que se están produciendo en una actualidad marcada por la globalización. En éste sostiene la idea de que asistimos a un fin de ciclo histórico, al final de un paréntesis, que se abrió en la ilustración y la modernidad, y que ahora se está cerrando, ya que el contexto del campo escolar se ha modificado profundamente. La educación reglada debe atender a las demandas del sistema económico y del mundo laboral. Ante la competencia internacional solamente cabe posicionarse bien en un marco competitivo que pasa por la cualificación del trabajo y por mecanismos de gobernanza. Si el capital va a la búsqueda de valor añadido y de productividad, éstos dependerán de la cualificación de la mano de obra. Razón por la cual es importante para capitales y empresas contar con una información útil y fiable sobre el valor comparativo de los títulos universitarios. De ahí la necesidad de un adecuado y objetivo sistema de indicadores que clasifique a las diferentes universidades. La internacionalización de la economía y la sociedad del conocimiento requieren rankings universitarios para adaptarse a los tiempos de la aceleración del cambio.

La universidad ya no es sólo un sujeto histórico (Durkheim, 1992], cuya soberanía descansaba en buena parte en el principio de autonomía, sino que ahora también se ha convertido también en un objeto heterónomo de interés social y de demandas externas en un contexto de marcada y creciente mercantilización del saber y del conocimiento. Esta dualidad estructural entre la educación como un derecho del sujeto y como un objeto productible y mercantilizable parece reproducir la dualidad de estatus del trabajo en las sociedades capitalistas: como derecho y como mercancía [Supiot, 2015], hasta el punto deque podemos conjeturar que el derecho a la educación ha sido enmarcado por la economía política de la educación. Las leyes y las políticas educativas parecen estar dejando paso a los algoritmos educativos, a las normas educativas. Sin disponer aun prácticamente del desarrollo de un sistema de indicadores que operacionalice el derecho a la educación [Tomasevski, 2004], nos hemos visto asediados por un sistema de indicadores [De Miguel et al. 2001: 127] que nos permite medir, comparar y clasificar a nuestros sistemas universitarios en relación a la excelencia.

Solamente hay que recordar el punto de partida de uno de los trabajos colectivos de referencia en el análisis de los cambios acaecidos en el sistema universitario: "el concepto de universidades de rango mundial ha sido firmemente adoptado en las políticas institucionales y gubernamentales para promover la competitividad en un mundo cada vez más globalizado. Sin embargo, la paradoja es que el concepto ha sido generalmente empleado sin una definición clara y explícita." [Wang, Cheng and CaiLiu 2012: 2]. La razón es simple, no se trata de un concepto, sino de un término utilizado retóricamente. Además, convendría no olvidar la útil diferenciación entre las nociones de ídolo, concepto e idea, para poder examinar el sentido específicamente ideológico que tiene la idea de "World Class Universities"[WCU].Algunos autores se refieren a la ubicuidad de la retórica de la excelencia, señalando que "excelencia es el estándar de oro del mundo universitario" (Moore et al. 2017: 2].Sin embargo, "entre eruditos, una de las aproximaciones comunes a la definición de WCU es por medio de la creación y el consecuente desarrollo de tablas, tales como ARWU, THE, QS World University Rankings. Buscando definir el término los estudiosos han identificado atributos clave que tienen las universidades "world-class" y que no poseen las universidades ordinarias [corrientes, habituales]" (Altbach 2004, 2011). Esta determinación se ha visto concretada en una serie de características relativas a abundancia de recursos, gobernabilidad favorable y concentración de talento [Salmi 2009]. Son estos conceptos comparativos los que nos permiten ir más allá de meros conceptos clasificatorios, pero no nos permiten elaborar tasas de crecimiento y establecer proporciones, propias de los conceptos métricos, definidos como una aplicación entre un sistema comparativo y la serie de los números reales (Pérez y Aldás, 2018]. 


\subsection{Observando sistemas de clasificación: ¿hacia una métrica universitaria?}

La palabra excelencia se esgrime ad nauseam como arma contra posibles competidores. [15]

Si hay algo común a las clasificaciones universitarias es que parecen muy pegadas a la realidad concreta, tratan de visibilizar los hechos más significativos de las universidades. Dicho de otro modo, comparten una epistemología decididamente empirista. La idea es atenerse a los hechos, asumir la transparencia universitaria por medio de una constatación de los hechos. El problema es que no podemos confundir los hechos concretos con los hechos científicos. ¿Establecen los rankings hechos científicos sobre las universidades? Un dispositivo instrumental, por perfeccionado que esté, no configura por sí un proceso científico de investigación. Requiere, a su vez, de una problemática y de un sistema teórico de conceptos que posibilite el establecimiento de ciertas relaciones que hagan inteligible al campo universitario. Suponer la existencia de un Sistema Universitario del que se pueden tomar medidas es, desde una perspectiva científica y sociológica, al menos cuestionable. ¿Por qué confiar en los hechos que se nos presentan, aunque sea a través de sofisticados instrumentos? En sentido estricto, los rankings nos dan "la apariencia" de la universidad, pero ¿nos ofrecen la estructura de las leyes de variación de las leyes específicas del sistema histórico social de las universidades?

La estructura científica de los conceptos usados en las clasificaciones no implica necesariamente la naturaleza científica de un proceso de investigación. Pasar de conceptos comparativos a conceptos métricos es sólo el primer paso para aproximarse a la realidad empírica. Pero el fin de la ciencia es establecer explicaciones, relaciones causales que van más allá de la descripción y, por tanto, mecanismos que nos pongan en una relación determinada las variables previamente construidas. El método científico debe ir de las abstracciones [conceptos] a las síntesis articuladas de las mismas [concretos de pensamiento], para evitar que dichas abstracciones sean resultado de una generalización de lo que se presenta empíricamente a nivel concreto [Marx, 2017). El problema siempre es separar el objeto real del objeto de conocimiento y los rankings, tanto internacionales como nacionales, ofrecen una determinada visión de un supuesto objeto real. La primacía del objeto matemático va en la dirección de que se trata de una mezcla entre lo sensible y lo inteligible. Mezcla que se ve reflejada en el principio de continuidad entre el saber de los expertos y el saber del sentido común. De ahí la popularidad de estas clasificaciones. No hay ruptura con el sentido común, con el saber inmediato de la conciencia. Solamente hay una diferencia de grado entre conciencias, siendo la supuesta cientificidad una especie de toma de conciencia especial.

\subsection{Universidades de rango mundial a través de rankings internacionales}

La perfección existe porque es imaginable, pero no es perfecta porque es inalcanzable. [4]

La aparición de los rankings mundiales de universidades como el Times Higher Education World University Rankings [THE], el Academic Ranking of World Universities (ARWU], o el QS World University Rankings [QS], además de ser un resultado de la globalización, como refieren Marope, Wells y Hazelkorn [2013], es una señal inequívoca de que las universidades viven realmente "en una época de mediciones y comparaciones".

Los rankings como el QS, el ARWU o el THE tienen una gran capacidad para sintetizar y proveer 
de datos duros sobre el estatus de los sistemas educativos y las universidades, tanto para el público en general como para los tomadores de decisiones de las Instituciones de Educación Superior [IES] [Erkkilä 2013). Esto facilita supuestamente la supervisión de los avances tanto de las políticas educativas como de las estrategias y los planes institucionales que se implementan en los diferentes niveles de la gestión educativa en el nivel superior. Los rankings varían según su orientación metodológica. El ARWU, por ejemplo, clasifica a las universidades manejando indicadores cuantitativos sobre la producción y el impacto de la investigación que realizan las universidades, mientras que otros -el QS o el THE- comparan a las IES tomando criterios de carácter más subjetivo como las encuestas de opinión sobre la reputación e imagen institucional de las universidades [Ordorika and Rodríguez 2010].

A pesar de las críticas que puedan hacerse en contra de los rankings - muchas de ellas fundamentadas, entre otras cosas, por su cuestionable construcción metodológica-, no se puede negar su aparente legitimidad [Altbach 2006], al ser propias de este nivel de lo social, ni el impacto que tienen en el ámbito universitario. Posiblemente uno de los efectos más destacables sea la poderosa influencia que ejercen "en las decisiones de los estudiantes sobre la universidad a la que deberían asistir, y cuanto estarían dispuestos a pagar" por un título de determinada institución [Ordorika and Lloyd 2013: 210].

De acuerdo con Marmolejo, los rankings se han convertido en parte del entorno institucional de la educación superior. Aunque es cierto que estos conceptos comparativos "pueden ser una herramienta útil que ayuda a guiar el mejoramiento institucional" en el nivel superior (Marmolejo 2010: 2], también lo es que deben analizarse con escepticismo y manejarse con cuidado, como suscribe Baty [2012], editor del ranking THE.

Sin duda, entre los rankings que han alcanzado mayor proyección e influencia a nivel mundial, destaca el Ranking Académico de las Universidades del Mundo (Academic Ranking of World Universities - ARWU). Este ranking fue publicado por primera vez en junio de 2003 por el Centro de las Universidades de Clase Mundial [Center for World-Class Universities-CWCU] de la Escuela Superior de Educación [anteriormente el Instituto de Educación Superior] de la Universidad Jiao Tongde Shanghái de China, y su periodicidad es anual. ARWU utiliza seis indicadores para clasificar las universidades del mundo. Estos indicadores son el número de alumnos y profesores que han ganado premios Nobel y medallas Fields, el número de investigadores altamente citados, el número de artículos publicados en revistas de Nature y Science, el número de artículos indexados en Science Citation Index-Expanded [SCIE] y Social Sciences Citation Index [SSCI], y el rendimiento per cápita respecto al tamaño de una institución. Califica más de 1.200 universidades al año y las 500 mejores se publican en la web.Un estudio publicado por The Economist en 2005 aludió a ARWU como el ranking anual más utilizado por universidades de investigación en todo el mundo. Ahora bien, para este ranking cada universidad es una especie de objeto del que se "miden" una serie de características singulares y arbitrarias.

\subsection{La complejización de las clasificaciones universitarias}

La excelencia acaba cristalizando en un conjunto de reglas tan rígido que la hace inmune frente al cambio con lo que la excelencia se hace cada vez menos excelente. [10] 
El papel de la ANECA [Bermejo, 2011] en relación con otras agencias similares de aseguramiento de la calidad en el panorama internacional es reflejo de un proceso complejo y dinámico de gobernanza epistémica que se ha ido configurando en el marco del Estado evaluador (Elliot 2002). No nos detendremos en los desarrollos de las políticas de calidad. Un examen de los mismos en Europa Occidental y con una perspectiva transnacional se ha llevado a cabo en diferentes contribuciones (Neave 1995; Lorenzo Quiles and Cruz de Gracia 2015]. El caso de España también se ha analizado con detalle en trabajos previos [Montané, Beltrán and GabaldónEstevan, 2017; Montané, Beltrán and Teodoro 2017].

Ante la diversidad de metodologías y de orientaciones, en 2013, una década después de la puesta en marcha de ARWU, se comenzó a elaborar en España el proyecto ISSUE (Indicadores sintéticos de las universidades españolas], que ha dado como resultado el U-Ranking, y cuya sexta edición se ha publicado en 2018. Entre los criterios seguidos para el desarrollo de ISSUE los autores destacan el siguiente: "Desarrollar múltiples rankings de las universidades, según se contemple la actividad universitaria desde una perspectiva general en una dimensión específica [docencia, investigación, innovación y desarrollo tecnológico] y según se haga desde la perspectiva del rendimiento alcanzado [U-Ranking] por cada universidad, pero también del volumen total de los resultados [U-Ranking volumen]. [Pérez y Alduz de Graciad, a [les" (Dewey,or tdaries, Auda en Accisexista recomats per la Unitat dHigher Education. A Global Perspective. ás 2017: 9].

La distinción entre un ranking de rendimiento y un ranking de volumen, que contemplan los indicadores sintéticos del proyecto ISSUE, no es trivial, pues tener en cuenta o no el tamaño de las universidades es relevante. Hacer una u otra opción no supone una carencia ni una ventaja desde el punto de vista metodológico, pero sí implica adoptar una perspectiva que debe tenerse en cuenta a la hora de presentar los resultados. Del mismo modo, si bien en algunos casos el rendimiento de los resultados puede ser más importante que el volumen de los mismos, en otros casos el tamaño puede ser relevante. Así, una universidad muy grande pero con un nivel de resultados muy pobre es un problema mucho mayor que una universidad con ese mismo nivel pero más pequeña. Pérez y Aldás justifican el interés de ambos enfoques y señalan como algunos de los rankings más citados [el de Shanghái sin ir más lejos] son de volumen, puesto que la mayoría de variables con las que se construye proporcionan datos absolutos [número de premios nobel o medallas Fields], pero no relativos al tamaño de la universidad. Otros rankings, como el QS World Universities Ranking, utilizan variables normalizadas por tamaño. Y otros contemplan ambas comparaciones, como hace el ranking I-UGR relativo a los resultados de investigación.

La diferencia con los anteriores es que el ISSUE combina ambas perspectivas (desplegadas en dos rankings generales] en tres dimensiones principales: docencia, investigación, e innovación y desarrollo tecnológico (dando lugar a dos rankings más específicos, denominados U-Ranking dimensiones y U-Ranking titulaciones]. En cada una de las dimensiones contempla los ámbitos referidos a recursos, producción, calidad, e internacionalización, con indicadores diferentes para cada uno de ellos. Otra peculiaridad del proyecto ISSUE, generador del U-Ranking, es que considera que la docencia y la investigación pueden tener una importancia distinta para cada usuario de los servicios de las universidades. A partir de esta consideración incorpora una herramienta web que permite elaborar rankings personalizados [a demanda) para tender las preferencias del usuario, dando lugar a lo que los autores denominan "análisis de sensibilidad".

Este análisis puede reflejar si los resultados de las universidades en docencia e investigación están correlacionados, porque si bien suele darse por supuesto que ambas dimensiones están 
correlacionadas, esta hipótesis no está suficientemente contrastada, y la mayor parte de los rankings priorizan la investigación. "Que la dimensión investigadora sea más fácil de medir no debería ser argumento para no medir la calidad de la docencia", señalan los autores [ibid:45]. A nuestro juicio, es este un argumento tan débil como común, pues lo que prima en definitiva es el interés de una dimensión (la investigadora] sobre otra (la docente), con efectos, como hemos señalado al principio, en la propia gobernanza epistémica y en la reconfiguración del modelo de universidad. La sobreevaluación de la investigación tiene efectos significativos, en términos de políticas y prácticas educativas, que desembocan en una devaluación o depreciación de la docencia. En las conclusiones, los autores señalan la importancia central que tiene combinar medidas de rendimiento investigador con rendimiento docente, para evitar sesgos de la realidad, ya que la correlación entre ambas es muy baja.

En general, el posicionamiento es directamente proporcional a los recursos movilizados en la educación superior. El Informe llevado a cabo permite concluir, con relación a España, por ejemplo, que su producción científica es adecuada al tamaño del país, pero que los recursos movilizados son sensiblemente menores a los de los países que ocupan las primeras posiciones; de hecho, menores incluso a lo que corresponde, comparativamente, a la riqueza del país. Y esta variable incide determinantemente en el posicionamiento en rankings.

En sus conclusiones, Pérez y Aldás [ibid: 67-70] constatan que las universidades públicas lideran el SUE, en especial las actividades de investigación y de innovación y desarrollo tecnológico. Como en la edición de 2016, las universidades catalanas encabezan los rankings de investigación [Pompeu Fabra], y de innovación y desarrollo tecnológico [Politécnica de Catalunya). La docencia está encabezada por la Universidad Politécnica de Valencia ex aequo con las universidades de Navarra, Deusto, Antonio Nebrija y Mondragón]. Y la Pompeu Fabra también encabeza el ranking de rendimiento.

Por otra parte, Pérez y Aldás constatan que si bien algunas iniciativas internacionales en este terreno muy conocidas —como el Ranking de Shanghái o el del Times Higher Education [THE]han aumentado la visibilidad de las clasificaciones de universidades y su demanda, estos rankings ponen el énfasis en los indicadores de investigación y en la formación de prestigio internacional, con frecuencia de posgrado, excluyendo la mayor parte de la actividad docente que no compite en las ligas mundiales. [lbid: 68].

Si bien los rankings son un instrumento legítimo y útil en muchos aspectos, uno de los principales problemas estriba en la facilidad que tienen para simplificar la realidad y crear la impresión de que una universidad es mejor que otra, basándose en una serie de indicadores usados arbitrariamente para medir la calidad de las universidades [Shin, Toutkoushian and Teichler 2011; Erkkilä 2013; Federkeil, Van Vught and Westerheijden 2012; Marope, Wells and Hazelkorn 2013; Ordorika and Lloyd 2013; Ordorika and Rodríguez 2010].

En la proliferación de rankings España ha jugado un papel importante, ya que varios de los rankings más prominentes a nivel mundial se han confeccionado en nuestro país. Así, "Webmetrics Ranking of Worls Universities" (Aguillo 2012], o el Scimago Institutions Ranking; a los que se puede añadir el Ranking I-UGR de Universidades Españolas por Campos y Disciplinas Científicas, ya que como indica Elías Sanz-Casado et al. [2013] la existencia de rankings nacionales "resulta esencial para las regiones y países no centrales." 
Sin duda, cabe reconocer el trabajo español para crear un sistema de indicadores integrado y combinado, más equilibrado y que corrige debilidades y sesgos de los rankings internacionales más conocidos. El instrumento presentado [ISSUE] es un intento de combinar y sintetizar la arquitectura de rankings españoles e internacionales, si bien no escapa a la "obsesión por la eficacia, por la eficiencia, por el recurso sistemático a metáforas del mundo de la producción, o por el discurso omnipresente de calidad, de evaluación, de los resultados y del rigor." [Santos, Teodoro and Da Costa Junior 2016: 35].

\subsection{Aspectos problemáticos de la ideología de la excelencia}

La creatividad es una vocación, la excelencia una reputación. [19]

Proponemos reflexionar sobrela necesidad de un cambio de perspectiva para atravesar el espejo de las apariencias. Las WCU actúan como un espejo en el que no importa tanto como se ven reflejadas las diferentes instituciones, sino que el punto crucial consiste en constatar que las instituciones son observadas y vigiladas bajo su mirada atenta y sancionadora.

Todo punto de vista consiste en tener la vista puesta en un punto poderoso, difuminando otros posibles modos de ver la realidad. Aquí queremos ofrecer otros puntos de vista, que cuestionan la mirada ciclópea y excluyente en la que se basa la jaula de hierro de las WCU.Llegando incluso a problematizar la noción de perspectiva y de punto de vista. El ojo de la excelencia no es ojo porque lo veamos, es ojo porque nos ve. Es decir, el espejo de la excelencia es una construcción social, cuyo diseño y patrón se ha propagado y en el que nos vemos reflejados, medidos, clasificados, observados y comparados. Pero aquí viene una significativa parte del problema,como es que se pretenda medir y simplificar de manera aritmomórfica (matematizable, cuantificable) aspectos complejos que forman parte de una realidad compleja y contradictoria (Montané, Beltrán and Teodoro 2017]. Calidad y excelencia son conceptos ambiguos, elusivos, polisémicos, de textura abierta; de ahí su naturaleza ideal e ideológica, nada incompatible con la noción de perspectiva, al contrario. Es más, podríamos hacer una distinción entre idea, que remite a visión y a delimitación, e ideal que se vincula con el manejo y lo ilimitado. Si la calidad es una idea, la excelencia es un ideal, pero ninguna es un concepto rigurosamente definido.

En lo que sigue señalaremos algunos aspectos problemáticos de la WCU que no conviene perder de vista:

a) Su elitismo.Al igual que la globalización, las WCU y los instrumentos de clasificación a su servicio han hecho que las universidades entren en una dinámica de competencia internacional que ha favorecido únicamente a algunas instituciones, generalmente de gran arraigo y prestigio histórico. Dicho de otra forma, los rankings han creado una dinámica de abundantes perdedores y pocos ganadores [Marginson 2012). Los rankings también son elitistas por naturaleza: más de 16.000 universidades en el mundo nunca podrán clasificarse en alguno de estos comparativos [Rauvargers 2011], debido a que no están orientadas hacia la producción de ciertos tipos de investigación.

b) Su relación con el auto-prestigio. Otro aspecto destacable es la utilización de determinados indicadores, por ejemplo, ARWU recurre al número de premios Nobel y medallas Fields obtenidas por los egresados y docentes. Puede cuestionarse, sugieren Federkeil, Van 
Vught y Westerheijden [2012], si este indicador en realidad mide la excelencia o mide el prestigio de las universidades para atraer el talento. Aquí se nos puede plantear la duda de la endogeneidad, al no saber si son prestigiosas por sus talentos o capturan talentos, porque son prestigiosas. La indecisión sobre esto implica dudas sobre el modelo, ya que no podemos determinar si se trata de producir "excelencia" o de adquirirla, apropiándose de recursos externos, en función de una cierta ordenación geográfica.

c] El monopolismo monolingüístico y cultural. Tomar en consideración el número de veces que son citadas las publicaciones de los investigadores como indicador de calidad representa otro problema, ya que los científicos norteamericanos que son los más numerosos tienden a citar a sus compatriotas, dejando de lado a un gran número de publicaciones producidas por investigadores de otras partes del mundo, en especial las de aquellos que no son anglófonos (Altbach, 2016). Esta cuestión provoca un efecto Mateo, que perjudica a las universidades de los países en donde el inglés no es la lengua oficial o usual.

c) Su vinculación con el gerencialismo: Hace dos décadas Stephen J. Ball ya advertía que el nuevo culto a la excelencia suponía la introducción de técnicas propias del mundo de los negocios y el "culto de la excelencia" en las instituciones del sector público [Alonso, 1996). El nuevo gerencialismo exige atención constante a la "calidad", cercana a las demandas del consumidor [Alonso y Fernández, 2013b]. En el terreno de la educación esto supone el desplazamiento de formas de organización profesionales por regímenes de organización empresariales[Ball 1998: 123]. Mucho más recientemente, algunos autores se refieren al problema de la fetichización de la excelencia y a sus raíces (Moore et al. 2017: 10).

d] Su individualismo competitivo. A juicio de Nelly Stromquist, en el entorno propio de las WCU, donde la competición es tan fuerte, las medidas cuantitativas se irán haciendo más rígidas y estandarizadas con el tiempo, pero dejando de lado cualquier medida alternativa o visión crítica que proponga otro tipo de resultados educativos, como aquellos orientados hacia la inclusión social o a la socialización y a la educación [Anaya, 2007]. El individualismo competitivo propio de las WCU no sólo mide la diferencia, sino que la construye [Stromquist 2017: 142). Una estrategia para ello tiene que ver con el llamado impacto de la producción científica. "La calidad de la investigación de la educación superior se evalúa sin tomar en cuenta el contenido de los libros, artículos o ponencias publicados por los investigadores [...] sino únicamente el número de impactos [es decir, de veces que ha aparecido su título], en ciertas revistas especializadas en la catalogación de estos índices de impactos" [Pardo, 2013: 284].

f] Producción de hegemonía. Las WCU son un reflejo de una poderosa hegemonía en educación superior. Esta hegemonía se lleva a cabo a través de la construcción de visiones dominantes [Gramsci, 2013] que delimitan un ámbito determinado y se nutren de conceptos y discursos aceptados. Un complejo proceso por el que las instituciones de los países más fuertes diseminan normas y criterios que se asumen como sentido común. En el ámbito de educación superior, esta operación se lleva a cabo, entre otros medios, a través de los rankings, que premian a aquellos que más se acercan al modelo "ideal", y que actúan como "harvard-ometros", que miden cómo una institución se asemeja a Harvard [Ordorika and Lloyd 2015: 392]. 
g] Una decidida ausencia de frónesis. Para Hans-Dietz Meyer hay aspectos sociales que no son susceptibles de clasificación: "Ordenarlos significaría cometer la falacia de la precisa imprecision, la cual es a menudo un camino para salvar la teoría mientras se hace desaparecer al fenómeno (Meyer 2017: 18]. Meyer acusa una notable ausencia de phronesis, es decir, de atributos propios de la esfera ética, en la proliferación de rankings que pretenden medir la educación desde una perspectiva únicamente cuantitativa. Desde esta perspectiva, las WCU incurren también en la falacia pitagórica del intelectualismo, que olvidan el valor inapreciable de la experiencia como vehículo de conocimiento, que ya destacó John Dewey en Democracy and Education ([1916] 1998]. Para Dewey, la experiencia no es cuantificable. ¿Qué tipo de información nos dan las WCU sobre las formas de vida de los investigadores, sus trayectorias académicas, su forma de relacionarse o de intercambiar prácticas, experiencias y conocimiento?

La ausencia de frónesis, en este caso, contrasta con el atributo que caracteriza a las WCU, el de la hybris, la desmesura (la pulsión de ofrecer resultados por encima del resto). La cualidad que reclamamos desde la comunidad educativa, en cambio, es mucho más sencilla: no busca tanto medir más sino más bien aquello que realmente importa y que nos concierne a todos como sujetos individuales y colectivos. El problema consiste en saber qué es.

De estos hechos y de sus contradicciones se toma conciencia a través de ciertas formas de conciencia, por medio de determinados sistemas de discursos que, aunque determinados socialmente, se presentan como autónomos y con capacidad de justificar los hechos y explicar las contradicciones

\section{LOS MITOS DE LAS WORLD CLASS UNIVERSITIES Y DE LAS PERFORMANCES: DIMENSIÓN IMAGINARIA DE LA IDEOLOGÍA ACADÉMICA}

Para poder realizar un análisis sociológico de los hechos y prácticas educativas universitarias de enseñanza y aprendizaje conviene comenzar por un estudio de las diversas situaciones y de los múltiples conjuntos de disposiciones que están a la base de dichas acciones e interacciones [Bourdieu, 2015]. Uno de los componentes fundamentales de los contextos en que se desenvuelven las prácticas está constituido por el sistema de los discursos [Conde, 2009] que los diferentes agentes tienen sobre las mismas y por el campo ideológico que posibilita e imposibilita determinadas fuerzas discursivas [Marx, 2017]. Consideramos que en el sistema universitario se desarrolla una determinada ideología propia de ese campo específico, como mostró Marx con su singular análisis sobre la ideología propia del sistema económico. Es verdad que tras la obra de K. Mannheim el concepto de "ideología" se apartó de la circulación sociológica en beneficio del de "perspectiva". Sin embargo, hay que agradecer al trabajo de Bourdieu [2012] que rehabilitase su uso, hablando de ideologías como instrumentos de dominación e incluso que lo articulara con el de perspectiva, complejizando a éste [Bourdieu, 2016]. En cierto modo, este trabajo es la aplicación de la propuesta de Bourdieu sobre el poder simbólico al sistema de las clasificaciones universitarias. Propuesta que continúa la línea iniciada por Durkheim y Mauss, como él mismo señala [Durkheim y Mauss, 1903) y que complementa el programa de investigación de B. Bernstein sobre la articulación entre principios de clasificación y principios de control en el análisis de las prácticas pedagógicas (Bernstein, 1990).

Nuestra reflexión tiene en cuenta el contexto de cambios profundos, de fracturas y de desigualdades 
crecientes que impactan en las Instituciones de Educación Superior. Además, da continuidad a una serie de trabajos previos, integrados en un proyecto de alcance global e internacional. En el primero de ellos [Beltrán and Montané 2015], se puso énfasis en la cuestión de la gobernanza. El siguiente trabajo se centró en el problema de la equidad (Beltrán, Montané and Gabaldón 2016). Y a continuación, se abordó el tema relativo a la calidad en relación con sus mecanismos de acreditación [Montané, Beltrán and Gabaldón 2017).

Con la noción de excelencia que abordamos ahora [a modo de síntesis de la etiqueta o logo WCU] se puede configurar una seriecuyas fases están compuestas por las nociones de gobernanza, equidad, calidad, excelencia y eficiencia. Y lo que nos interesa no es tanto la comprensión lógica de estas representaciones sociales, sino su interpretación socio-lógica e histórica, teniendo en cuenta que el objeto de esa interpretación, en tanto que científica y crítica, tiene que ser el concepto de "relación".

\section{a. Excelencia en relación con Gobernanza}

La obsesión por la excelencia corre el riesgo de conducir hacia el pensamiento único, desde donde el riesgo es resbalar hacia el pensamiento nulo. [3]

En el primero de los trabajos mencionados, se estableció una conexión directa entre gobernanza y autonomía, señalando que la universidad está cada vez más condicionada por los requerimientos de la llamada "economía del conocimiento". [Beltrán and Montané 2015: 98-99]. Por otra parte, Stephen Ball ya advirtió del papel relevante de los modos de medir la educación en la actualidad, asociando la gobernanza educativa a la tiranía de los números [Ball 2015: 299-301]. En ese sentido compartimos la hipótesis de "gobernanza epistémica", según la cual asistimos a una gobernanza que opera sobre las concepciones de la realidad de los sujetos, afectando a las relaciones de poder en los modos de crear, gestionar y aplicar el conocimiento. [Alasuutari and Qadir 2016: 633-652]. Una gobernanza "sostenida a través de una nueva imagen de la geopolítica internacional del conocimiento" (Montané, Beltrán and Teodoro 2017: 284). Como veremos, el papel de los rankings para establecer estándares de excelencia y distinción universitaria (en términos de clase, en este caso, a nivel mundial] juega un papel predominante, hasta el punto de que lo que comenzó siendo un instrumento de información se ha convertido en un instrumento de prescripción con efectos materiales en las estructuras institucionales y en la agencia de los sujetos.

\section{b. Excelencia en relación con Equidad}

Un sistema educativo puede presumir de excelencia si está pensado para formar en ella a todos sus alumnos, pero no si se deshace de todos aquellos alumnos que no la alcanzan por sí mismos. [1]

En nuestro segundo trabajo, dedicado a la equidad en Educación Superior, advertíamos acerca de "la emergencia de una poderosa industria de indicadores asociada con una suerte de imperialismo aritmético". [Beltrán, Montané and Gabaldón-Estevan 2016: 150-151). De modo que el término equidad como sustituto de la noción de igualdad de oportunidades puede contribuir a difuminar, disminuir o a otorgar un significado "blando" a la constatación de una brecha cada vez mayor en términos de riqueza y pobreza, de asimetrías crecientes en la distribución de recursos y de 
concentración de oligopolios siguiendo la lógica de acumulación propia de un sistema económico capitalista. Pero ahora, se avanza un paso más porque las aspiraciones hacia la excelencia por parte de las instituciones universitarias, como objetivo prioritario, suponen una inversión radical respecto de la matriz histórica de la universidad y de sus ideales ilustrados, y acaban derogando cualquier tipo de pretensión igualitaria.

Además, la equidad es un corrector de las decisiones justas, al tiempo que consiste en dar a cada uno lo que merece. Con la equidad, la justicia debe atender a las circunstancias de los casos concretos. No se puede evaluar un sistema sin tener en cuenta sus méritos y condiciones. Los jueces, en este caso los expertos educativos, tendrán que adaptarse a la realidad de las universidades siempre que eso sea posible. El pilotaje de la maquina universitaria debe practicar la flexibilidad de la norma. Por eso no se pueden introducir principios materiales sustanciales relativos a la igualdad, sino principios formales funcionales que tengan en cuenta la equidad.

\section{c. Excelencia en relación con Calidad}

No por mucho insistir con la palabra excelencia se gana uno el derecho a identificarse con ella. [6]

Como ya habíamos sostenido en las conclusiones de un trabajo anterior (Montané, Beltrán and Gabaldón-Estevan, 2017: 61), los requisitos de calidad son, paradójicamente, establecidos por instrumentos y procedimientos hegemónicos principalmente cuantitativos, que terminan en una poderosa industria contable de indicadores, clasificaciones y comparaciones. Esta forma de medir la educación se desplaza, así, desde una lógica institucional con propósitos educativos hacia nuevas lógicas que subordinan el conocimiento y la formación a lo que se ha llamado capitalismo académico. Los instrumentos que sirven para el crecimiento productivo (Naredo 2006: 160] se han desarrollado en una medida mucho mayor que los instrumentos asociados principalmente con el desarrollo social o la ciudadanía [Anaya 2007: 65].El uso o la falta de instrumentos alternativos de medición y herramientas apropiadas tienen un gran impacto en la configuración y desarrollo de ciertas políticas, ya que facilita o dificulta este desarrollo. Y lo que es peor, la autonomía de lo numérico evidencia el primado de los medios sobre los fines, con las consecuencias políticas de ello.

Las relaciones entre estas palabras-clave revelan la necesidad de tener en cuenta las condiciones bajo las cuales se utilizan, la distribución y recepción de su uso y las connotaciones de su significado [esto es, su significado no explícito] en contextos determinados.

Aunque algunos estudios han señalado el efecto de refracción de estos "supuestos hechos concretos" a los que remiten estas nociones[Rudd and Goodson 2017], echamos en falta un programa de investigación que construya un adecuado sistema conceptual que logre explicar por qué se nos aparecen estos hechos de la gobernanza, equidad, calidad y excelencia como se nos aparecen y por qué se refractan en las conciencias como lo hacen. Un programa que problematice el sentido común y la naturalización de los fenómenos sociales. Aplicada al caso de las WCU, esta naturalización se basa en la convicción de que existe una "objetividad tecnocrática" sustentada en los rankings, a la que hay que doblegarse.

Es innegable que las universidades, en su intento de alcanzar la excelencia de las WCU, están siendo atrapadas en la jaula de hierro [nueva cultura, nuevo entorno, nueva tecnocracia) que 
están construyendo para conseguir ese objetivo, dejando en un segundo plano el principio político de autonomía en el que se desarrollaron históricamente [Durkheim, 1992]. El logo de WCU se está convirtiendo en una poderosa factoría generadora de grandes expectativas. Pero conviene recordar que el tamaño de la expectativa guarda correspondencia con el tamaño de su frustración.

\section{d. Excelencia en relación consigo misma y con Eficiencia}

La excelencia es un gran argumento cuando se demuestra en uno mismo y un viejo truco cuando solo se nombra para dudar de ella en los demás." [5].

Es verdad que la excelencia remite, en primer término, a una forma suprema de bien, dotándose asi de una cierta naturaleza ética. Sin embargo, el bien de una acción es su fin y éste difiere para cada actividad, de modo que la excelencia será algo distinto en la docencia, en la investigación y en la innovación. La excelencia debería ser, pues, un fin que no puede ser ya medio de ninguna otra cosa, el fin último o perfecto. Ahora bien, ¿cuál es el fin propio de la docencia?, ¿y el de la investigación?, ¿y el de la innovación? Si algo positivo tiene la noción de excelencia es que nos sitúa de lleno en la problemática de los fines sustanciales de la universidad. Y si algo negativo tiene la de rendimiento es que nos devuelve al ámbito de los medios: su dimensión positivista no puede ser más clara, a pesar de la apariencia sistémica.

La virtud de una actividad cualquiera es la excelencia propia de esa actividad. El problema estriba en definir o ponerse de acuerdo en las virtudes universitarias. La virtud de la docencia, de la investigación y de la innovación, ¿cuáles son?, ¿cómo definirlas? Una universidad que forma, investiga e innova bien es una buena universidad, posee la eficiencia o excelencia propias de la universidad, pero ¿qué es formar bien? [Anaya, 2007], ¿una buena y excelente universidad sería aquella en la que se enseña bien o se aprende bien? La excelencia no es más que una virtud, que se identifica con la eficiencia en el desempeño de una actividad. De ahí que consideremos que, a pesar del giro epistemológico protagonizado por el ISSUE, estemos en el mismo paradigma de lo que se le exige a la universidad. Ésta tiene supuestamente tres funciones y según las realice bien o no, poseerá las correspondientes eficiencias o excelencias. En cada función puede conseguirse la excelencia, esto es, la eficiencia. De ahí que haya una extraña continuidad entre medir las excelencias de las universidades y ordenar a las universidades excelentes. Por eso uno de los objetivos del sistema ISSUE es comprobar la correspondencia de sus resultados con las clasificaciones internacionales. De este modo, se trabaja en la dirección de lograr más fiabilidad y validez para los sistemas de indicadores que utilizan.

¿Qué se consigue con esta continuidad entre excelencia y eficiencia? Pensamos que hacer creer a los diferentes agentes sociales que la universidad es un mecanismo gobernable, una especie de máquina o dispositivo que sólo requiere de una buena dirección y control. El mítico discurso actual sobre la universidad es el que prima la gestión [Alonso y Fernández, 2013a). Considerar que el sistema universitario es un sistema de producción donde el valor es resultado del trabajo no es más que un medio para afirmar que la productividad depende de la correcta gobernabilidad. Todo puede reducirse a una mera cuestión técnica, a una flexible calculabilidad. De los sujetos a los algoritmos, podríamos decir. El dominio de los hombres y de las leyes parece haber sido sustituido por el dominio de las cosas y de las normas, lo cual refuerza el fetichismo y nos introduce en la magia de los números, es decir, en una especie de mística. Si las leyes de la Universidades son arbitrarias y políticas, el sistema universitario empieza a tener cierta entidad y autonomía respecto al resto de sistemas sociales que forman su entorno, porque podemos tomar medidas 
de él, ya que su lenguaje está escrito con caracteres matemáticos. El fin perseguido es claro: los hombres ya no gobiernan a los hombres, tampoco las leyes, son los números quienes gobiernan a los seres humanos. Ese es uno de los más habituales mecanismos de violencia simbólica.

Si los primeros sistemas de clasificación comienzan separándose de la organización de la estructura social, los actuales sistemas de clasificación contribuyen a presentar de forma autónoma a la propia organización. Así, la identificación progresiva de los modos de organización y de legitimación logra su doble meta; hacer incuestionable la estructura social que da pie a la organización y dificultar la problematización de la organización y de la legitimación. Por otra parte, la evolución de las modalidades de gobernanza, desde la equidad a la eficiencia ponen de manifiesto dos tendencias fundamentales: la decisiva lucha por la desigualdad, entronizada como valor esencial, y la sustitución no tanto de los principios de legitimación como de su forma. Pasar de una forma basada en la unidad a otra edificada sobre la multiplicidad es alejar a los sistemas educativos universitarios de su lucha por las formas de igualdad y de democratización. El primado de la multiplicidad diluye la cuestión de los fines sustanciales de la universidad. Todo se reduce a una cuestión de medios y a una geometría variable de relaciones "medios-fines", en la que reina la noción de "utilidad".

\section{LA MISTIFICACIÓN DE LOS RENDIMIENTOS UNIVERSITARIOS: LA DIMENSIÓN SIMBÓLICA DE LA IDEOLOGÍA ACADÉMICA}

La excelencia como imposición inicial dificulta la excelencia como resultado final. [11]

Es cierto, como señala el profesor Fernández Enguita que vivimos tiempos de encrucijada en la actualidad, marcados por la incertidumbre, la perplejidad y la insignificancia [Castel, 2010). Pero no es menos cierto que al mismo tiempo se nos ofrecen determinados dogmas y certidumbre [Naredo, 2006] que se nos presentan como autoevidentes. ¿Qué Estado o Nación no quieren ver aparecer a una de sus universidades en esos listados de prestigio que contribuyen de forma tan decidida al crecimiento económico y al desarrollo social? Sin duda asistimos en el presente a una crisis generalizada y multidimensional [Castells, 2016], pero también a una compleja serie de cambios culturales cuya función primordial es metaestabilizar el orden social y su estructura, neutralizando la crítica del conocimiento y de la sociedad (Alonso, 1996]. El desarrollo creciente de contradicciones corre parejo a la configuración de un nuevo orden simbólico que se presenta como ídolo, idea e ideal, como trascendente y, a la vez, inmanente, pero en todo caso incuestionable. Un orden que contribuye a configurar lo real que se nos presenta como falso, ya que nos imposibilita ver el hecho de que no veamos.

La sociología, como señala Medina Echavarría [2009] puede ayudarnos, con su disposición comprensiva, a darnos cuenta de la configuración de fuerzas que afectan a nuestras biografías, estructuras sociales y momentos históricos. No obstante, no podemos perder de vista que también la sociología es una fuerza simbólica, que contribuye a configurar y definir lo social y lo problemático de lo social. La sociología puede realizar una función mistificadora, como la que llevan a cabo los sistemas de clasificación universitarios. De hecho, es demostrable el entrecruzamiento de ambas actividades. Y puesto que la sociología puede realizar una función ideológica, se precisa una ciencia social de las ideologías frente a las ideologías de la ciencia. Las sociedades del capitalismo histórico han entremezclado lo ideológico y lo científico. Si en un primer momento lo ideológico devino científico, asistimos en la actualidad a una ideologización de lo científico, proceso 
que tiene mucho que ver con la matematización de lo real, con su formalización, con el abandono de las cuestiones de contenido y con el alejamiento de todo lo relativo a el cuestionamiento de los contenidos. Por ello, ya no es suficiente con separar ideología de sociología. Ante la ideologización de lo ideológico solo cabe el camino de la sociología de la sociología, el camino de la crítica, que tan buenos resultados dio en los proyectos kantiano y marxiano. Hoy es necesario reformular la crítica de las racionalizaciones universitarias y poner las bases para una crítica de la economía política de las universidades. El problema de los procesos mistificadores es que siempre son tautológicos, no explican, y siempre apuntan al mismo fin, a la diferenciación, a la división, a la desigualdad. Son formas de justificar el sistema de las desigualdades sociales, el sistema, en este caso, de las distinciones entre universidades. Todos los rankings que hemos analizado comparten la intencionalidad de poder dotar a la gestión universitaria de un capital simbólico, del que al parecer carece. Sabido es que esta forma de capital es muy diferente a las demás, ya que es otorgado por el otro, no se posee por sí. La proliferación y complejización de los rankings es un paso más en el camino de hacer del capital simbólico algo impuesto a los diversos sujetos sociales. Un paso más en el proceso de apropiación de los recursos.

Si queremos utilizar un símil deportivo, es como si en una carrera de ciclismo distinguiéramos con claridad a los primeros en llegar a la meta, seguidos a continuación por un "pelotón" o masa de ciclistas en los que resulta muy difícil apreciar las diferencias entre quienes llegan casi a la vez. El símil deportivo sirve de nuevo como metáfora en la que se da a entender la doble característica, competitiva y olímpica, de las WCU. Se trata de una carrera sin fin que persigue ganar o ser el primero. Pero si solo reconocemos este modelo, olvidamos que existen alternativas posiblemente más inteligentes por cooperativas. La inteligencia social sigue siendo una tarea pendiente. Y más cuando las actividades que trabajan por ella son devaluadas y en el límite no tenidas en cuenta por el cálculo de las utilidades. La paradoja de todo este proceso es que se valora lo que carece de efectivo valor de uso, haciendo sacrosanto un inefable valor de cambio que sólo oculta una imposición arbitraria.

Por otra parte, no se puede hacer abstracción del significado profundo e implícito que encierra la propia noción de universidades de "rango" mundiales. La naturalización del concepto de clase aplicada a la educación superior supone algo más que la legitimación de las desigualdades en el ámbito universitario. Pues ahora ya no se trata sólo de aceptar la existencia de estas desigualdades, sino más bien de participar en su construcción con el argumento de perseguir la excelencia y la eficiencia. La desigualdad queda así recontextualizada como excelencia o distinción de clase y, lejos de ser considerada parte del problema, se percibe como parte de la solución. Muy atrás queda el principio de igualdad de oportunidades que sirvió de guía y horizonte a las universidades en el contexto del capitalismo de consumo.

El marco lógico que preside la presencia de universidades de rango mundial cambia definitivamente las reglas del juego y nos sitúa en un modelo diferente: los fines de la universidad ya no están orientados por los "ideales practicables" [Dewey[1916] 1998] de la ilustración entendida como emancipación ni de la justicia como forma de vida social. Los fines nobles que servían de horizonte a una universidad que se reclamaba a sí misma como autónoma han sido sustituidos por objetivos cortoplacistas y productivistas, al servicio de la demanda de clientes, consumidores o grupos de interés, ya sean estos estudiantes, empresas o Estados. El carácter democrático de la buena educación, entendida como bien común (Mattei, 2013), ha quedado sustituido por la invocación a la mejor educación, un privilegio de clase solo al alcance de una minoría. 
En su significado más profundo y radical, la prioridad por la excelencia educativa -en términos de clase, la defensa de los privilegios de una elite social que ahora se pretende mundial- supone la negación de cualquier objetivo igualitario y emancipador para la educación y la asunción definitiva de la necesidad lógica e inmutable de la desigualdad social. Considerada como verdad, la excelencia -frente a la igualdad- se convierte en un dogma; considerada como realidad, la excelencia se convierte en un mecanismo de regulación y presión transnacional; considerada como aspiración común, la excelencia se convierte - parafraseando a Dewey- en un "ideal impracticable" [por definición, la excelencia para todos es una contradicción en los términos: no todos pueden ser los mejores]; considerada en su dimensión política, frente al carácter democrático de la igualdad, la excelencia tiene un carácter aristocrático; considerada como metáfora, la excelencia es un espejismo que distorsiona la realidad, un nuevo "ídolo" (en el sentido baconiano) del lenguaje.Por eso, en coincidencia con las conclusiones de una reciente reflexión, "necesitamos un profundo cambio de perspectiva para recuperar la dimensión social y reconstruir los discursos sociales de los actores que participan y están implicados en las propias instituciones." [Montané y Beltrán, 2018: 60).

Una lección que Alicia aprendió en el País de las Maravillas es que no importa lo que signifiquen las palabras, sino quien es el dueño de las mismas. Por eso, necesitamos reapropiarnos de las palabras para empezar a elaborar una nueva práctica discursiva que, teniendo en cuenta las voces del mundo social, sea capaz de derogar la distinción de clase que caracteriza a las WCU y nos encamine hacia la construcción de una universidad ciudadana, plural y comprometida. Esa universidad ya no será un espejo que haya que atravesar, sino un cristal en el que mirarnos y reconocernos como iguales en la diversidad, una experiencia común para cooperar en la tarea creativa, radical y siempre por hacer, de promover la igualdad y la democracia sociales. Para ello debemos romper la mistificación de los números e ir más allá de las formas de conciencia, rompiendo con ellas por medio de determinados sistemas conceptuales que nos permitan comprender y explicar las posibilidades y límites del campo universitario desde su estructura y funcionamiento, desde su entorno complejo y su sistema de fines definible colectivamente.

\section{REFERENCIAS BIBLIOGRÁFICAS}

Aguillo, Isidro F. [2012]. Rankings de universidades: El ranking web. Higher Learning Research Communications. 2 [1], pp. 3-22. Disponible en http://dx.doi.org/10.18870/hlrc.v2i1.56

Alasuutari, Pertti andAli Qadir [2016]. "Imageries of the social world in epistemic governance" en International Sociology, 31 [16], 2016, pp. 633-652.

Alonso, Luis Enrique (1996) "El discurso de la privatización y el ataque a la ciudadanía social” en Cuadernos de Relaciones Laborales,8, pp.39-67.

Alonso, Luis. E. y Fernández, Carlos J. [2013a] "Los discursos del Management. Una perspectiva crítica". Lan Harremanak, 28, pp.42-69.

Alonso, Luis. E. y Fernández, Carlos J. [2013b] Los discursos del presente, Madrid, SXXI.

Altbach, Philip G. [2004] "The cost of benefits of world-class universities". Academe, 90 [1], pp. 20-23. 
Altbach, Philip G. [2006] “The Dilemmas of Ranking”. En Altbach, Philip [ed.], International higher education: Reflections on policy and practice, USA Chesnut Hill,pp. 77-80, disponible en www. bc.edu/content/dam/files/research sites/cihe/pubs/Altbach 2006 Intl HigherEd.pdf

Altbach, Philip G. [2016]Global Perspectives on Higher Education. Baltimore: John Hopkins University Press.

Anaya, Gonzalo(2007)La passió educativa. Valencia, Universitat de València.

Ball, Stephen, J. [2015]: "Education, governance and the tyranny of numbers" en Journal of Education Policy, 30 [3], pp.299-301.

Ball, Stephen J. [1998] "Big Policies/Small World: An introduction to international perspectives in education policy" en Comparative Education, 34:2, pp. 119-130, disponible en https://doi. org/10.1080/03050069828225

Baty, Phil. [2012]Rankings don't tell the whole story-Handle them with care. University World News. Disponible enhttp://www.universityworldnews.com/article. php?story=20120626171938451

Beltrán, José and Alejandra Montané [2015] "Higher Education in Spain: Framework for Governance and Autonomy in Spanish Universities", en Joshi, K. M. and Saeed Paivandi [eds.].Global Higher Education: Issues in Governance,. New Delhi, B. R. Publishing House,pp. 63-103

Beltrán, José, Alejandra Montané and Daniel Gabaldón-Estevan [2016] "Higher Education in Spain: Framework for Equity", en PAIVANDI, Saeed and K.M. JOSHI. [eds.].Equity in Higher Education. A Global Perspective, New Delhi, Studera Press,pp. 149-185.

Bermejo, José C. [2011]La maquinación y el privilegio, Madrid, Akal.

Bernstein, Basil [1990]Poder, educación y conciencia, Barcelona, El Roure.

Bourdieu, Pierre [2012]“Sobre el poder simbólico” en Intelectuales, política y poder, Buenos Aires, Eudeba, pp. 71-80.

Bourdieu, Pierre [2015] Sociologie Générale Vol. 1, Paris, Raisons d’agir/Seuil.

Bourdieu, Pierre [2016] Sociologie Générale Vol. 2, Paris, Raisons d’agir/Seuil

Castel, Robert [2010] El ascenso de las incertidumbres, México: FCE.

Castells, Manuel [2016]De la crisis económica a la crisis política. Una mirada crítica, Barcelona, La Vanguardia.

Conde, Fernando, [2009] Análisis sociológico del sistema de discursos, Madrid, CIS, Cuadernos metodológicos nำ 43 .

De Miguel, Jesús M., Caïs, Jordi y Vaquera, Elizabeth [2001] Excelencia. Calidad de las universidades españolas, Madrid, CIS.

Dewey, John [1916] [1996] Democracia y Educación, Madrid, Morata. 
Domingos Sobrinho, Moisés; Ennafaa, Ridha y Chaleta, Elisa [2016] La educación superior, el estudiantado y la cultura universitaria, Valencia, Neopatria.

Durkheim, Émile et Mauss, Marcel. [1903] "De quelques formes primitives de classification". Année sociologique, 6. Disponible en http://bibliotheque.uqac.ca/index.htm

Durkheim. Émile [1895] [1974] Las reglas del método sociológico, Madrid, Morata.

Durkheim, Émile [1904-5] [1992] Historia de la educación y de las doctrinas pedagógicas, Madrid, La Piqueta.

Echavarría, José Medina (2009) Responsabilidad social de la inteligencia. Estudios nuevos sobre nuestro tiempo. Madrid, FCE.

Elliot, John [2009] "La reforma educativa en el Estado evaluador". Perspectivas, vol. XXXII, n. 3, September. Disponible en URL http:/ /firgoa.usc.es/drupal/files/elliots.pdf

Erkkilä, Tero [2013] Global University Rankings: Challenges for European Higher Education. New York, Palgrave Macmillan.

Federkeil, Gero; Frans A. van Vught and Don F. Westerheijden [2012] "An Evaluation and Critique of Current Rankings".En F. A. van Vught and F. Ziegele [Eds.], Multidimensional Ranking. The Design and Development of U-Multirank, pp. 39-70. Netherlands: Springer Science+Business Media B.V. doi: 10.1007/978-94-007-3005-2

FernándezEnguita, Mariano[2016] La educación en la encrucijada, Madrid, Fundación Santillana.

Georgios, Stamelos; K. M. Joshi and Saeed Paivandi [eds.].(2017) Quality Assurance in Higher Education.A Global Perspective. New Delhi: Studera Press.

Gramsci, Antonio [2013] Antología, Madrid, Akal.

Joshi, K. M. and Saeed Paviandi [eds.].(2015) Global Higher Education: Issues in Governance. New Delhi, B. R. Publishing House.

Lorenzo Quiles, Oswaldo and Efrain Cruz de Gracia [2015] "Calidad y evaluación de la Educación Superior. Una perspectiva transnacional a través de los rankings" en DEDiCA. Revista de Edução e Humanidades, 8, pp. 155-174.

Marginson, Simon [2012] "Global University Rankings: The strategic issues". En Las Universidades Latinoamericanas ante los Rankings Internacionales: Impactos, Alcances y Limites. UNAM, 17 y 18 de mayo, p. 17. México, D.F. Disponible en http://www.encuentrorankings.unam.mx/ Documentos/ConferenciaMagistralMarginsontexto.pdf

Marmolejo, Francisco [2010]The Madness of Rankings. The Chronicle of Higher Education, disponible en http:/ / chronicle.com/blogs/worldwise/the-madness-of-rankings/27100.

Marope, Priscilla Toka Mmantsetsa; Peter J. Wells and Ellen Hazelkorn, eds. [2013)Rankings and accountability in Higher Education: Uses and Misuses, UNESCO.

Marx, Karl [2017] El Capital, Madrid, SXXI. 
Mattei, Ugo [2013] Bienes comunes, Madrid, Trotta.

Meyer, Heinz-Dieter [2017]"The limits of measurement: misplaced precision, phronesis, and other Aristotelian cautions for the makers of PISA; APPR, etc." en Comparative Education, 53 [1], pp. 17-34. Doi: 10.1080/03050068.2017.1254981

Montané, Alejandra; José Beltrán and Daniel Gabaldón-Estevan [2017] "Higher Education in Spain. Framework for Quality Assurance", en Georgios, Stamelos;K.M. Joshi, and Saeed Paivandi [eds.].Quality Assurance in Higher Education. A Global Perspective, Delhi, India, Studera Press,pp. 41-67.

Montané, Alejandra; José Beltrán and António Teodoro [2017] “La medida de la calidad educativa: acerca de los rankings universitarios" en RASE, 10 [2], pp. 283-300.

Montané, Alejandra y Beltrán, José [2018] "Rankings en educación superior: reflexiones y escenarios de la calidad educativa" en Gionara Tauchen y Altair Alberto Fávero [orgs]. Avaliação doensino superior. Perspectivas mundiais, Curitiba, editorial CRV, pp. 43-64.

Moore, Samuel; Cameron Neylon; Martin Paul Eve; Daniel Paul O. Donnelland Damian Pattinson [2017] 'Excellence R Us': university research and the festishisation of excellence" en Palgrave Communications, 19 Jan. Doi: 10.1057/placomms.2016.105

Naredo, José M. [2006] Raíces económicas del deterioro ecológico y social, Madrid, SXXI.

Neave, Guy [1995)“Las políticas de calidad: desarrollos en Enseñanza Superior en Europa Occidental". Revista de Educación, 308, pp.7-29.

Ordorika, Imanol and Marion Lloyd [2013]"A decade of international university rankings: a critical perspective from Latin America". En Marope, Priscilla Toka Mmantsetsa; Peter Wells and Ellen Hazelkorn, [eds.].Rankings and accountability in Higher Education: uses and misuses, Paris, UNESCO Publishing,pp. 209-232. Disponible en http://unesdoc.unesco.org/ images/0022/002207/220789e.pdf

Ordorika, Imanol and Lloyd, Marion [2015] "International rankings and the contest for university hegemony". Journal of Educational Policy, 30 [3], pp. 385-405. Disponible enhttp://dx.doi.org /10.1080/02680939.2014.979247

Ordorika, Imanol and Roberto Rodríguez [2010] "El ranking Times en el Mercado del Prestigio Universitario". Perfiles Educativos, vol. XXXII [129],pp. 8-29.

Paivandi, Saeed and K. M. Joshi, K. M. [eds.]. [2016].Equity in Higher Education. A Global Perspective, New Delhi, Studera Press.

Pardo, José Luis [2013]. "El conocimiento líquido. Sobre la reforma de las universidades públicas” en Jesús Hernández, Álvaro Delgado-Gal y Xavier Pericay [eds.]. La universidad cercada. Testimonios de un naufragio, Barcelona, Anagrama, pp. 267-292.

Pérez, Francisco and Aldás, Joaquín [dirs.] [2017]U-Ranking 2017. Indicadores sintéticos de las universidades españolas. Instituto Valenciano de Investigaciones Económicas, Fundación BBVA, junio [quinta edición], doi: http:/ / dx.medra.org/10.12842/RANKINGS_SP_ISSUE_2017 
Pérez, Francisco and Aldás, Joaquín [dirs.][2018]U-Ranking 2018. Indicadores sintéticos de las universidades españolas, Fundación BBVA e Ivie, disponible en https://www.fbbva.es/wpcontent/uploads/2018/06/Informe-U-Ranking-FBBVA-Ivie-2018.pdf

Ramas, Clara [2018] Fetiche y mistificación capitalistas, Madrid, SXXI.

Rauhvargers, Andrejs. 2011. Global University Rankings and Their Impact. Brussels: European University Association, disponible en http://www.eua.be/pubs/Global_University_Rankings_ and_Their_Impact.pdf

Rudd, Tim and Ivor Goodson [2017] Negotiating Neoliberalism. Developing Alternative Educational Visions,Rotterdam, Sense Publishers.

Salmi, Jamil [2009] El desafío de crear universidades de rango mundial, Colombia, Banco Mundial en coedición con Mayol Ediciones.

Santos, Eduardo; AntónioTeodoro and Reinaldo Da Costa Junio[2016] "Rankings Universitários: entre a regulaçao do mercado e a difusao de modelos organizacionais - o caso brasileiro" en Del Vecchio, Angelo and Eduardo Santos [orgs.] Educaçao Superior no Brasil; Modelos e Missoes Institucionais, Sao Paulo, BT Academica,pp. 33-55.

Sanz-Casado, Elías et al. [2013] "Rankings nacionales elaborados a partir de múltiples indicadores frente a los de índice sintético". Revista española de documentación científica, 36 [3], disponible en doi: http://dx.doi.org/10.3989/redc.2013.3.1.023

Shin, Jung Cheol; Robert K. Toutkoushian and Ulrich Teichler[eds.][2011]University Rankings: Theoretical Basis, Methodology and Impacts on Global Higher Education,Netherlands, Springer Science + Business Media B.V.

Stromquist, Nelly P. [2017] “The professiorate: the challenged subject in US higher education”. Comparative Education, 53 [1]. Doi: 10.1080/03050068.2017.1254975

Supiot, Alain [2015] La gouvernance par les nombres, Paris, Fayard.

Tomasevski, Katarina[2004]Indicadores del derecho a la educación, disponible en http:// www.derechoshumanos.unlp.edu.ar.

Trías, Eugenio [1975] Teoría de las ideologías, Barcelona, Península.

UNESCO [2015] Rethinking Education. Towards a Global Common Good? Paris, UNESCO.

Wagensberg, Jorge [2017] "Sobre la existencia y unicidad del método científico" en Wagensberg, J. Teoría de la creatividad, Barcelona, Tusquets, pp. 213-245.

Wang, Qi, Ying Cheng and Nian Cai Liu [eds.] [2012] Building World-Class Universities. Different Approaches to a Shared Goal, Rotterdam, Sense Publishers. 